\title{
Parameter identification and state-of-charge prediction of decommissioned lithium batteries
}

\author{
Qian Shi ${ }^{1, \mathrm{a}}$, Junkai Li ${ }^{2}$,, Qi Qin ${ }^{3, \mathrm{c}}$ and Chijian Zhang ${ }^{4, \mathrm{~d}^{*}}$ \\ ${ }^{1}$ School of Physics and Electronic Information, Anhui Normal University, Wuhu, Anhui, 241000, China \\ ${ }^{2}$ Anhui Vocational College Of Defense Techology, Luan, Anhui,237011, China \\ ${ }^{3}$ School of Physics and Electronic Information, Anhui Normal University, Wuhu, Anhui ,241000, China \\ ${ }^{4}$ School of Physics and Electronic Information, Anhui Normal University, Wuhu, Anhui ,241000, China
}

\begin{abstract}
Aiming at the problem that different temperatures and working modes affect the parameter identification and state of charge (SOC) estimation of decommissioned lithium batteries, a new method based on the second-order RC equivalent circuit model combined with the recursive least square method (RLS) is proposed to introduce the forgetting factor, and combined with the extended Kalman filter algorithm (EKF) to realize the method of online parameter identification of decommissioned lithium batteries and the optimal estimation of SOC. In order to solve the problem of obtaining the optimal solution of the error covariance matrix and the measurement noise covariance matrix in EKF, the particle swarm optimization algorithm (PSO) is used to optimize online to further improve the SOC prediction accuracy. The results show that the joint optimization algorithm can accurately identify the parameters and SOC values of retired lithium batteries, which is helpful to realize the echelon utilization of retired lithium batteries.
\end{abstract}

\section{Introduction}

With the rapid development of new energy vehicles, when the performance of lithium power batteries drops to $80 \%$ of the original performance, they will not be able to meet the standards for electric vehicles. Therefore, a large number of retired lithium batteries will be produced every year. Retired lithium power batteries contain a large amount of heavy metals and organic matter, toxic gases produced by electrolytes and their conversion products, which will seriously threaten the environment and human health, and cause huge pressure on the social and ecological environment. Therefore, recycling and cascading lithium power batteries not only create economic value for society, but also reduce waste discharge for society, which is a major ecological construction task. Lithium-ion batteries are relatively active, and the internal characteristics of the battery will change under the influence of ambient temperature and working mode, especially the internal parameters of decommissioned lithium batteries change more obviously. Therefore, a sophisticated battery parameter identification and SOC prediction model is established for decommissioning. The echelon utilization of lithium batteries is of great significance [1].

At present, there are many methods for parameter identification and SOC prediction of lithium batteries. Literature [2] proposed a lithium battery SOC estimation method based on parameter identification and adaptive extended Kalman filter algorithm (AEKF). This method can achieve better accuracy and convergence speed under simulated conditions, but ignores the noise coordination. The automatic optimization process of the optimal solution of the variance matrix adds a certain amount of tasks. Literature [3] proposed the use of rolling time domain algorithm (MHE) combined with EKF algorithm, using the idea of rolling time domain window, integrating the window information of the previous $\mathrm{N}$ moments to estimate the current SOC value, by establishing an arrival function and adding an $\mathrm{M}$ estimator instead The measurement error effectively improves the estimation accuracy, but the time complexity is high. Literature [4] proposed a staged lithium-ion battery state-of-charge estimation method. According to the working state of the battery system, the estimation process is divided into the initial stage, the middle stage of charge and discharge, and the later stage of charge and discharge. Different methods are used for different stages. The relevant parameters in the ampere-hour integral formula can be corrected and compensated, although it can improve the accuracy and stability of the estimation, it is not practical.

Based on the advantages and disadvantages of the above research, and considering the battery temperature and SOC application characteristics, this paper conducts research and analysis on the performance of decommissioned lithium batteries under different working conditions, and establishes a second-order RC equivalent circuit model that can truly simulate decommissioned lithium. The internal dynamic characteristics of the battery, and the accuracy of the model are verified by exponential fitting of the actual measured voltage and comparison of

d*Corresponding author's e-mail: whzcj_cn@sina.com 
the identification results. In order to further improve the identification results and the accuracy of the SOC prediction, this paper adopts EKF and the RLS algorithm with forgetting factor. The joint method is used to estimate the SOC value, and the initial values of the error covariance matrix and the measurement noise covariance matrix in the EKF are globally optimized online through PSO to obtain the optimal parameters.

\section{A battery equivalent circuit model based on the forgetting factor recursive least square method}

The dynamic circuit model of the decommissioned lithium battery established in this paper is shown in Figure 1. This model is a second-order RC model. According to the voltage characteristics of the decommissioned lithium battery in the actual working process, the dual RC inertia link is used to simulate the internal polarity of the lithium battery. The slow rise of voltage caused by chemical reaction [5]. Among them: Uoc means open circuit voltage, $\mathrm{U}_{\mathrm{L}}$ means terminal voltage, $\mathrm{I}_{\mathrm{L}}$ means current, $\mathrm{R}_{0}$ means ohmic resistance, $\mathrm{R}_{1}$ means concentration polarization resistance, $\mathrm{R}_{2}$ means electrochemical polarization resistance, $\mathrm{C}_{1}$ means concentration polarization capacitance, $\mathrm{C}_{2}$ means polarization capacitance .

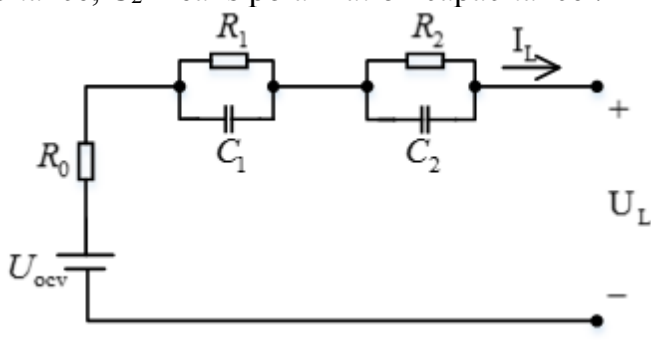

Figure 1. Battery equivalent circuit model

According to Kirchhoff's law, the expression of the model can be obtained.

$$
\left\{\begin{array}{l}
U_{\mathrm{L}}=U_{\mathrm{oc}}[\operatorname{SOC}(t)]-U_{1}-U_{2}-I(t) \cdot R_{0} \\
C_{1} \cdot \frac{d U_{1}}{d t}=I(t)-\frac{U_{1}}{R_{1}} \\
C_{2} \cdot \frac{d U_{2}}{d t}=I(t)-\frac{U_{2}}{R_{2}}
\end{array}\right.
$$

The SOC value of the battery can be obtained by the ampere-hour integration method, and $Q_{n}$ represents the actual capacity of the battery.

$$
\operatorname{SOC}\left(t_{1}\right)=\operatorname{SOC}\left(t_{0}\right)-\frac{\int_{t_{0}}^{t_{1}} I(t) d t}{Q_{n}}
$$

In order to improve the accuracy and speed of parameter identification, the correction function of RLS is used to correct the estimated value at the previous time through the new measured data, so as to obtain the estimated value at the current time to realize online parameter identification. Although the RLS algorithm is simple and stable, with the increase of data, the correction ability will decrease, which will affect the accuracy of identification. Therefore, the forgetting factor $\lambda$ is introduced into the algorithm to increase the impact of new data on the system, so that the algorithm can respond quickly and converge to near the actual value. By adjusting the value of $\lambda$, the initial value of the covariance matrix of the recursive least squares method is updated to calculate the gain matrix $\mathrm{K}_{0}$. Then update the parameters to be estimated at time $\mathrm{k}$. Finally, the values of parameters $\mathrm{R}_{0}, \mathrm{R}_{1}, \mathrm{R}_{2}, \mathrm{C}_{2}$, and $\mathrm{C}_{2}$ in the equivalent circuit model are identified.

$$
\begin{gathered}
P_{O}(k)=\frac{1}{\lambda}\left[I-K_{O}(k) \Phi(k)^{T}\right] P_{O}(k-1) \\
K_{O}=\frac{P_{O}(k-1) \Phi(k)}{\lambda+\Phi(k)^{T} P_{O}(k-1) \Phi(k)} \\
\theta(k)=\theta(k-1)+K_{O}(k)\left[U_{L}(k)-U_{O C}(k)-\theta(k-1)^{T} \Phi(k)\right]
\end{gathered}
$$

\section{SOC prediction of decommissioned lithium battery based on PSO-EKF}

\subsection{Principle of SOC prediction based on EKF algorithm}

The EKF algorithm is mainly to linearize the nonlinear system. The higher-order term is omitted through Taylor series expansion, and only the first-order term is retained, and the state-space equation of the nonlinear system is linearized [6,7]. The linearized system state equation and observation equation are as follows.

$$
\left\{\begin{array}{l}
x(k+1)=A(k) \cdot x(k)+B(k) \cdot u(k)+w(k) \\
y(k)=C(k) \cdot x(k)+D(k) \cdot u(k)+v(k)
\end{array}\right.
$$

Among them, the parameters in the equation are as follows

$$
\left\{\begin{array}{l}
A(k)=\frac{\partial f(x(k), u(k))}{\partial x(k)} \mid(x(k), u(k)) \\
B(k)=\frac{\partial f(x(k), u(k))}{\partial u(k)} \mid(x(k), u(k)) \\
C(k)=\frac{\partial g(x(k), u(k))}{\partial x(k)} \mid(x(k), u(k)) \\
D(k)=\frac{\partial g(x(k), u(k))}{\partial u(k)} \mid(x(k), u(k))
\end{array}\right.
$$

In the formula, $x(k+1)$ is the state matrix of the system at time $k+1, y(k)$ is the output variable of the system, $u(k)$ is the input variable of the system, $w(k)$ and $v(k)$ are the system process noise and observation noise, respectively, and $\mathrm{Q}$ and $\mathrm{R}$ respectively represent their covariance. The system corrects the predicted value of the state variable and the covariance matrix according to the actual output value to obtain the optimal filter value [8]. 
Based on the second-order RC equivalent circuit model, this paper takes current and terminal voltage as the input variable $u$ and output variable $y$ of the EKF, and the SOC, $U_{1}$, and $U_{2}$ of the decommissioned lithium battery as the state variables of the EKF. Then the linear discrete state space equation of the battery model is obtained.

$$
\begin{aligned}
& \left\{\begin{array}{l}
x(k+1)=A \cdot x(k)+B \cdot u(k) \\
y(k)=C \cdot x(k)+D \cdot u(k)
\end{array}\right. \\
& \left\{\begin{array}{l}
x(k)=\left[S O C(k) U_{1}(k) U_{1}(k)\right]^{T} \\
u(k)=I(k) \\
y(k)=U_{L}(k)
\end{array}\right.
\end{aligned}
$$

$$
A=\left[\begin{array}{ccc}
1 & 0 & 0 \\
& e^{-\frac{T}{\tau_{1}}} & 0 \\
0 & 0 & e^{-\frac{T}{\tau_{2}}}
\end{array}\right] \text {, }
$$

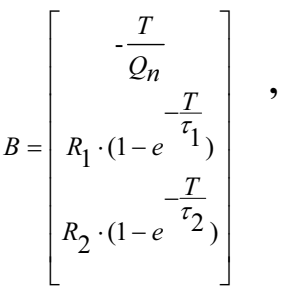

$$
\begin{aligned}
C= & {\left[\begin{array}{lll}
\frac{U_{O C}(S O C(k))}{S O C(k)} & -1 & -1
\end{array}\right], \quad D=-R_{0}, } \\
& \tau_{1}=R_{1} \cdot C_{1}, \quad \tau_{2}=R_{1} \cdot C_{2}, \quad \mathrm{~T} \text { is the sampling }
\end{aligned}
$$
period。

When the values of $\mathrm{A}, \mathrm{B}, \mathrm{C}$, and $\mathrm{D}$ are determined, online joint estimation of model parameters can be performed according to the EKF algorithm. EKF has good adaptability. If the initial value error is large, the state can be adjusted by adjusting the noise variance $Q$ and $R$. The variable finally converges to near the true value after multiple iterations, which makes the algorithm more robust. When $\mathrm{Q}$ takes a smaller value or $\mathrm{R}$ takes a larger value, the gain will be smaller, which will weaken the correction effect of the actual value on the predicted value [9], so the values of $\mathrm{Q}$ and $\mathrm{R}$ need to be adjusted continuously. However, the manual adjustment range is large, it takes a long time, and the effect is difficult to achieve the ideal.

\subsection{Use PSO to optimize EKF noise matrix}

PSO is a biological evolutionary algorithm. Each particle in the population represents a parameter that needs to be optimized in the algorithm, corresponding to a fitness value determined by the fitness function [10]. In this paper, the absolute error between the predicted value of the prediction equation and the actual value is used as the fitness value, and then the position and velocity of the particles are updated according to the fitness value. The update process of particle velocity and position in standard PSO is as follows.

$$
\begin{gathered}
v_{i d}(k+1)=\omega * v_{\mathrm{id}}(k)+c_{1} r_{1}\left(p_{\mathrm{id}}(k)-x_{\mathrm{id}}(k)\right)+c_{2} r_{2}\left(p_{\mathrm{gd}}(k)-x_{\mathrm{id}}(k)\right) \\
x_{\mathrm{id}}(k+1)=x_{\mathrm{id}}(k)+v_{\mathrm{id}}(k+1) \\
\omega=\omega_{\max }-\left(\omega_{\max }-\omega_{\min }\right) * \mathrm{t} / \mathrm{T}_{\max }
\end{gathered}
$$

In the formula, $\mathrm{i}=1,2 \ldots, \mathrm{m} ; \mathrm{c} 1, \mathrm{c} 2$ are learning factors; $\mathrm{r} 1, \mathrm{r} 2$ are random numbers between $[0,1] ; \omega$ is the constant of inertia; $\mathrm{k}$ is the number of times; $\mathrm{t}$ and $\mathrm{T}_{\max }$ are the number of iterations and the maximum iteration, respectively frequency.

In order to simplify the calculation, the parameters to be optimized are combined into a vector vector.

$$
\varepsilon=(Q, R)^{\mathrm{T}}
$$

\section{Algorithm verification and analysis}

The experiment selected a decommissioned lithium iron phosphate battery with a nominal voltage of $3.2 \mathrm{~V}$ and a nominal capacity of $2.7 \mathrm{Ah}$ as the object, and the hybrid pulse power Characteristic (HPPC) was performed on the battery under test at a high temperature of $60^{\circ} \mathrm{C}$ to verify the feasibility of the PSO-EKF algorithm. This mode mainly simulates the working conditions of battery charging-discharging-shelving-discharging to test the pulse ability of the battery, and the pulse ability is essentially the change of the battery internal resistance. Different temperatures, currents, etc. will cause the battery polarization internal resistance to change Therefore, the HPPC mode can be used as a method to consider the health of the battery, which provides value for the echelon utilization and safety research of decommissioned lithium batteries. The data acquisition platform is shown in Figure 2 , which mainly collects data such as battery terminal voltage, terminal current, and SOC.

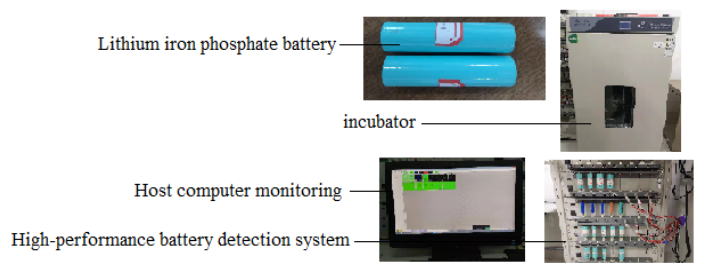

Figure 2. Data acquisition platform

Figure 3 shows the result of PSO optimization of EKF noise matrix under $60{ }^{\circ} \mathrm{C}$ high temperature HPPC conditions, population size $\mathrm{M}=30$, learning factor $\mathrm{c} 1=\mathrm{c} 2=2$, number of iterations $\mathrm{T}_{\max }=50$, maximum inertia weight $\omega_{\max }=0.9$, and minimum inertia weight $\omega_{\min }=0.4$.

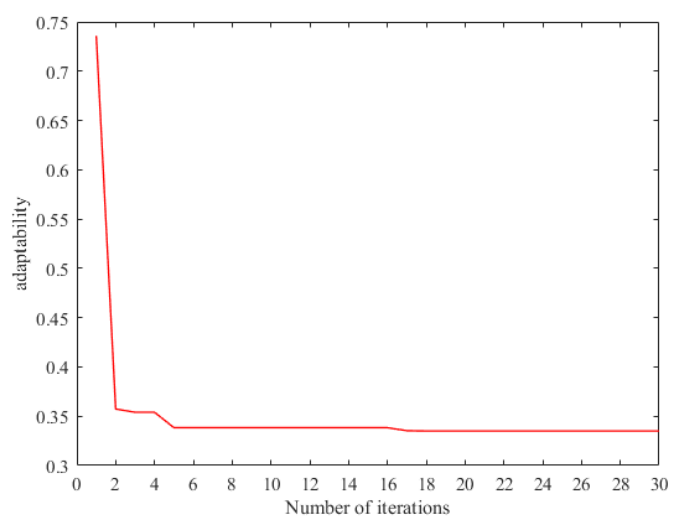

Figure 3. Number of iterations and optimal solution 
It can be seen from Figure 3 that the global optimal fitness value is obtained after 5 iterations and tends to be stable. As the number of iterations increases, the fitness value gradually decreases, and finally a stable optimal solution is obtained.

Figure 4 shows the parameter identification results of decommissioned lithium batteries. Figure 5 shows the comparison between the not optimized EKF noise matrix and the PSO algorithm optimized EKF noise matrix. Figure (a) shows the comparison of the SOC prediction results. It can be seen from the figure that it is not optimized The algorithm has a large error at the beginning of discharge and when the discharge SOC is lower than $40 \%$, and it cannot track well. The optimized result of the PSO algorithm can effectively overcome the interference of noise, which is basically close to the measured value, and has better tracking performance.

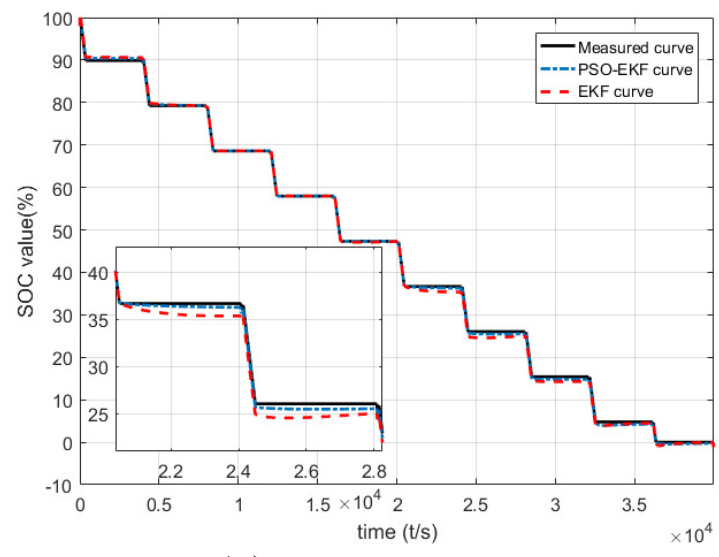

(a) SOC forecast results
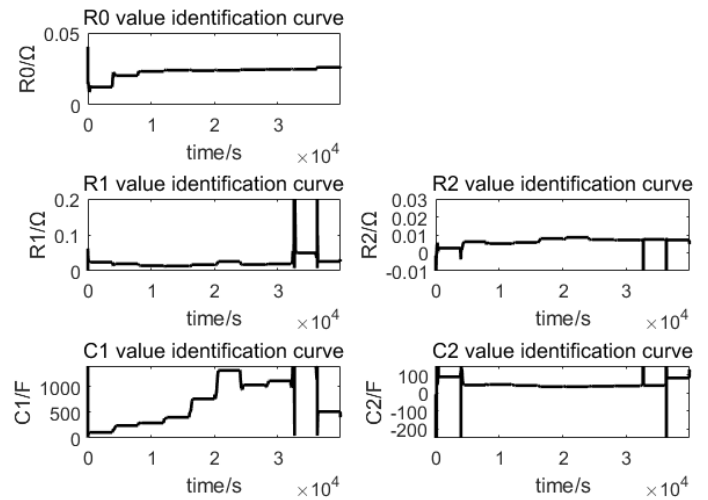

Figure 4. Parameter identification results

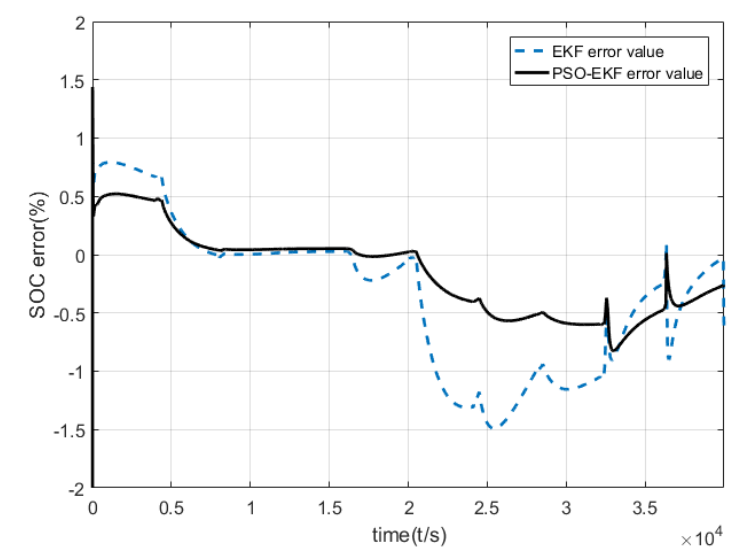

(b) Error comparison

Figure 5. Algorithm comparison

Figure (b) is the error comparison. It can be seen that the optimized error is significantly smaller than the not optimized error. It can be seen from Table 1 that the maximum error between the not optimized algorithm and the true value is up to $1.5 \%$, while the maximum error of the SOC optimized by the PSO algorithm is only $0.8 \%$. Through comparison, it can be seen that the EKF model based on the PSO algorithm can more accurately estimate the decommissioned lithium The SOC value of the battery further improves the accuracy of the SOC estimation.

Table 1. Algorithm comparison under HPPC conditions

\begin{tabular}{|c|c|c|}
\hline Parameter & Average error & Maximum error \\
\hline EKF & 0.53 & $1.53 \%$ \\
\hline PSO-EKF & $0.3 \%$ & $0.8 \%$ \\
\hline
\end{tabular}

\section{In conclusion}

This paper proposes a PSO-EKF method that can accurately identify the parameters of the decommissioned lithium battery model and estimate the SOC value. The results show that, compared with the not optimized EKF filter algorithm, the SOC estimation accuracy of the PSOEKF filter algorithm is higher. After the algorithm converges, the average error is only $0.3 \%$, and it has a better tracking ability. It laid the foundation for the echelon utilization of retired lithium batteries, and at the same time realized "turning waste into treasure", which greatly improved the utilization rate of resources. It conforms to the concept of sustainable development, realizes the use of clean energy and the re-creation of value, and is of great significance to the construction of ecological civilization.

\section{Fund Project}

Key Science and Technology Project of Anhui Province (1804a09020099) 


\section{References}

1. I. U. Khalil, M. Ahsan, I. Ullah, A. Adnan, N. Khan and S. Nawaz, "SOC prediction of Lithium-Ion Battery using Extended Kalman Filter," 2018 International Symposium on Recent Advances in Electrical Engineering (RAEE), Islamabad, Pakistan, 2018, pp. 1-5.

2. X. Liu et al., "Online identification of power battery parameters for electric vehicles using a decoupling multiple forgetting factors recursive least squares method," in CSEE Journal of Power and Energy Systems, vol. 6, no. 3, pp. 735-742, Sept. 2020.

3. Li P, Wang H, Xing Z, et al. Joint estimation of SOC and $\mathrm{SOH}$ for lithium-ion batteries based on EKF multiple time scales[J]. 2020.

4. D. A. Pola et al., "Particle-Filtering-Based Discharge Time Prognosis for Lithium-Ion Batteries With a Statistical Characterization of Use Profiles," in IEEE Transactions on Reliability, vol. 64, no. 2, pp. 710720, June 2015.

5. Xu Y, Hu M, Fu C, et al. State of Charge Estimation for Lithium-Ion Batteries Based on TemperatureDependent Second-Order RC Model[J]. Electronics, 2019, 8(9):1012-.

6. Tian Y, Lai R, Li X, et al. A combined method for state-of-charge estimation for lithium-ion batteries using a long short-term memory network and an adaptive cubature Kalman filter[J]. Applied Energy, 2020, 265.

7. Wang Houlian,Zhou Gongbo. State of charge prediction of supercapacitors via combination of Kalman filtering and backpropagation neural network[J]. IET Electric Power Applications,2018,12(4).

8. Aung H , Low K S , Soon J J . State-of-charge estimation using particle swarm optimization with inverse barrier constraint in a nanosatellite[C]// Industrial Electronics \& Applications. IEEE, 2015.

9. Chandra Shekar A, Anwar S . Real-Time State-ofCharge Estimation via Particle Swarm Optimization on a Lithium-Ion Electrochemical Cell Model[J]. Batteries, 2019, 5(1).

10. Mao X, Song S, Ding F, et al. SOC Estimation of Lithium Battery Based on IPSO-BP Neural Network[J]. Journal of Physics: Conference Series, 2020, 1684(1):012152 (6pp). 